\title{
Benchmarking shielding simulations for an accelerator-driven spallation neutron source
}

\author{
Nataliia Cherkashyna* and Douglas D. DiJulio \\ European Spallation Source ESS AB, P.O. Box 176, SE-221 00 Lund, Sweden \\ Tobias Panzner, Emmanouela Rantsiou, and Uwe Filges \\ Paul Scherrer Institute, 5232 Villigen PSI, Switzerland \\ Georg Ehlers \\ Quantum Condensed Matter Division, Oak Ridge National Laboratory, \\ Oak Ridge, Tennessee 37831, USA \\ Phillip M. Bentley \\ European Spallation Source ESS AB, P.O. Box 176, SE-221 00 Lund, Sweden \\ and Department of Physics and Astronomy, Uppsala University, SE-751 20 Uppsala, Sweden
}

(Received 15 June 2015; published 19 August 2015)

\begin{abstract}
The shielding at an accelerator-driven spallation neutron facility plays a critical role in the performance of the neutron scattering instruments, the overall safety, and the total cost of the facility. Accurate simulation of shielding components is thus key for the design of upcoming facilities, such as the European Spallation Source (ESS), currently in construction in Lund, Sweden. In this paper, we present a comparative study between the measured and the simulated neutron background at the Swiss Spallation Neutron Source (SINQ), at the Paul Scherrer Institute (PSI), Villigen, Switzerland. The measurements were carried out at several positions along the SINQ monolith wall with the neutron dosimeter WENDI-2, which has a well-characterized response up to $5 \mathrm{GeV}$. The simulations were performed using the Monte-Carlo radiation transport code Geant 4 , and include a complete transport from the proton beam to the measurement locations in a single calculation. An agreement between measurements and simulations is about a factor of 2 for the points where the measured radiation dose is above the background level, which is a satisfactory result for such simulations spanning many energy regimes, different physics processes and transport through several meters of shielding materials. The neutrons contributing to the radiation field emanating from the monolith were confirmed to originate from neutrons with energies above $1 \mathrm{MeV}$ in the target region. The current work validates Geant 4 as being well suited for deep-shielding calculations at accelerator-based spallation sources. We also extrapolate what the simulated flux levels might imply for short (several tens of meters) instruments at ESS.
\end{abstract}

DOI: 10.1103/PhysRevSTAB.18.083501

PACS numbers: 28.20.Cz, 28.41.Ak, 28.41.Qb

\section{INTRODUCTION}

Accelerator-driven pulsed-spallation neutron source facilities have substantial shielding requirements for both safety and scientific purposes. At these types of facilities, neutrons are generated via interactions between a highenergy $(\mathrm{GeV})$ proton beam with a heavy metal target. This liberates large quantities of neutrons from the metal at a wide range of energies which can reach up to the primary beam energy [1]. The energies of interest for scientific investigations are in the meV-eV energy range, so the

\footnotetext{
*nataliia.cherkashyna@esss.se

Published by the American Physical Society under the terms of the Creative Commons Attribution 3.0 License. Further distribution of this work must maintain attribution to the author(s) and the published article's title, journal citation, and DOI.
}

neutrons are made to scatter in moderators placed close to the target position. At the same time, a significant fraction of the neutrons escape the target-moderator assembly and continue into the bulk shielding and the beamport entrances. These neutrons can lead to the appearance of background signals in the neutron scattering instruments, located some tens of meters away from the target station. While detectors on the instruments, and the majority of reactor survey meters, tend to have a very low response above a few $\mathrm{MeV}$, energetic neutrons can however just as easily scatter inelastically anywhere in the facility and liberate more neutrons if they have enough energy or thermalize in the instrument shielding, walls, air, and floor. In particular, at a pulsed-spallation source, experiments measuring across the frame boundary (i.e., when the next proton pulse arrives) may reveal a spike of "prompt" neutrons [2,3]. For these reasons, spallation neutron 
sources require extensive shielding which is very different and more substantial compared to that of a nuclear reactor $[1,4,5]$. At the same time, the bulk shielding represents a substantial fraction of the cost for such a facility, which ultimately takes away from the potential resources which could be available for the scientific instruments. The ability to accurately model and simulate radiation penetration through the bulk shielding is thus key for enhancing both instrument backgrounds and cost savings. For this purpose, the accumulation of experimental data at existing spallation facilities plays a central role in the benchmarking of popular radiation transport codes.

Of particular interest for the current work is the suitability of the Geant4 [6] radiation transport code for accelerator-driven spallation neutron source shielding calculations. Geant 4 has a number of attractive features that are described in Sec. IV A. At the European Spallation Source (ESS) [7], which is currently under construction in Lund, Sweden, Geant4 is employed in a number of different applications, which include the detailed simulation of instrument detectors and the design of shielding structures throughout the facility. This is in a large part due to the introduction of technologies from the realm of high-energy physics, in particular from laboratories such as European Organization for Nuclear Research (CERN) [8], into the neutron scattering community and also due to the continuously increasing beam energies which are reaching energy scales more traditionally familiar to high-energy physicists. For example, the Materials and Life Science Experimental Facility [9] at the Japan Proton Accelerator Research Complex (JPARC) [10] uses a proton beam of $3 \mathrm{GeV}$ energy and the proton beam energy at ESS will be $2 \mathrm{GeV}$.

In the current work, we present the results of measurements carried out at the Swiss Spallation Neutron Source (SINQ) [11] at the Paul Scherrer Institute, Villigen, Switzerland, in order to understand the neutron background leaking from the SINQ monolith. For the current work, we define the monolith as the entire target structure and surrounding shielding components. The measurements are compared to Geant 4 simulations of the spallation source. Furthermore, the calculated flux at the edge of the monolith is used to estimate the background signal at a generic short instrument at ESS. It should be mentioned that this contribution only represents a single component of the background signal.

\section{SWISS SPALLATION NEUTRON SOURCE (SINQ)}

SINQ is an accelerator-based spallation neutron source which is primarily designed for neutron scattering investigations. It is a continuous source with a flux of about $10^{14} \mathrm{n} / \mathrm{cm}^{2} / \mathrm{s}$ and provides beams of both thermal and cold neutrons for scientific studies. SINQ is situated at the end of a cascade of three accelerators that deliver a proton beam of

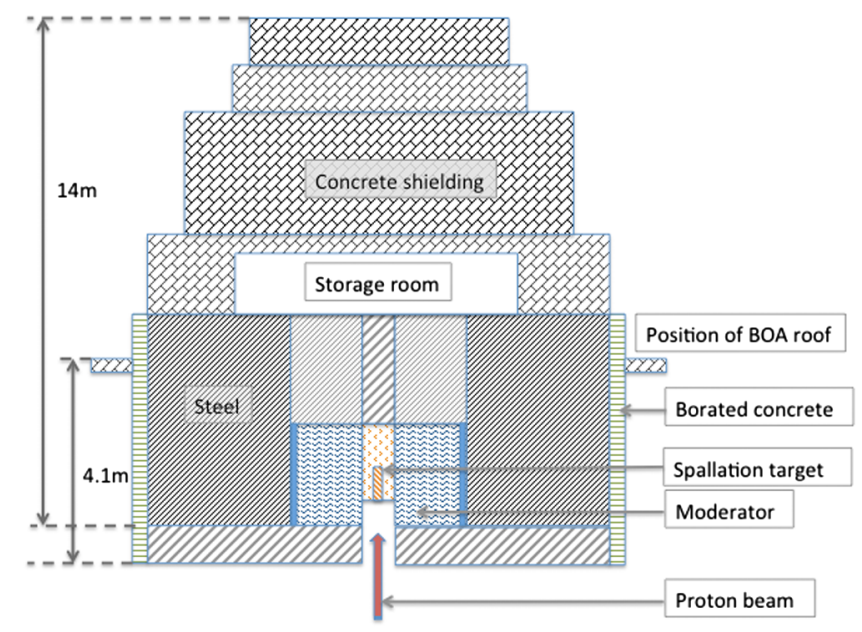

FIG. 1. Schematic drawing of SINQ model in Geant4.

$590 \mathrm{MeV}$ at a current up to $2.3 \mathrm{~mA}$. Below the SINQ-target station, the proton beam is diverted by bending magnets vertically upwards from underneath the ground, and directed onto a heavy metal target, which is an array of lead rods enclosed in zircalloy tubes and cooled with heavy water.

The target station contains a number of components. The target is surrounded by a $\mathrm{D}_{2} \mathrm{O}$ moderator, $2 \mathrm{~m}$ in diameter, and a $10 \mathrm{~cm}$ thick layer of $\mathrm{H}_{2} \mathrm{O}$. The target shielding consists of approximately $4.5 \mathrm{~m}$ of steel followed by $30 \mathrm{~cm}$ of borated concrete [12]. To reduce the skyshine of the radiation around the facility, due to the vertical diversion of the proton beam, several blocks of concrete are stacked on top of the steel shielding. The total height of the monolith is $14 \mathrm{~m}$ in the forward direction of the proton beam. Figure 1 highlights the important components for the simulations described below.

\section{NEUTRON BACKGROUND MEASUREMENTS AND ANALYSIS}

To understand the neutron background leaking out of the monolith, measurements were performed using the extended range neutron dosimeter WENDI-2 [13]. The WENDI-2 dosimeter consists of a $22 \mathrm{~cm}$ diameter polyethylene cylinder, loaded with a $1.5 \mathrm{~cm}$ thick tungsten powder shell, surrounding a ${ }^{3} \mathrm{He}$ detector. Fast neutrons can induce spallation reactions or undergo scattering processes in the tungsten layer and thus the dosimeter has an increased sensitivity at energies up to $5 \mathrm{GeV}$, in comparison to only $\sim 20 \mathrm{MeV}$ for a similar ${ }^{3} \mathrm{He}$ detector with polyethylene alone.

To perform the measurements with the WENDI-2 dosimeter along the monolith wall, the dosimeter was loaded into a wooden box attached to a crane. The measurements were carried out at various positions, about 1-2 meters in separation, along the monolith wall above the Beamline for Neutron Optics and Other Approaches (BOA) 


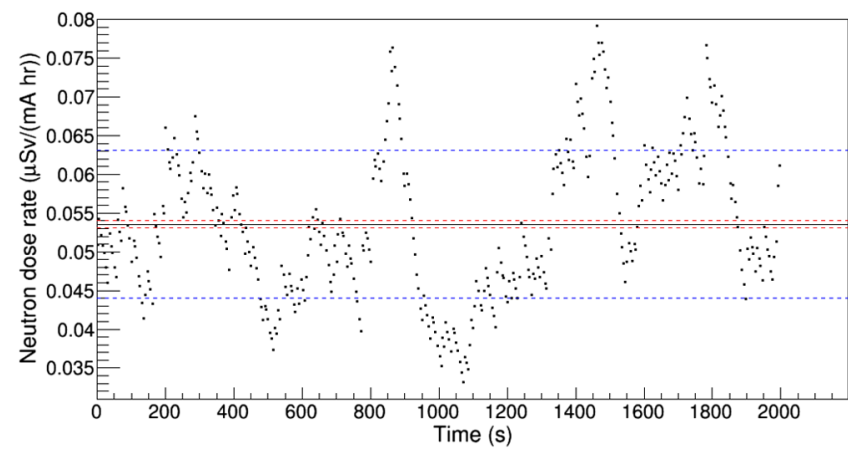

FIG. 2. Example of the data analysis for the measurements collected with the WENDI-2 dosimeter. The solid line represents the average of the data, the outer dashed lines represent the standard deviation and the inner dashed lines represent the standard deviation of the average.

[14] instrument cave. Each measurement position lasted for approximately $30 \mathrm{~min}$ and the average proton beam current was $1.5 \mathrm{~mA}$. Readout of the WENDI-2 dosimeter was performed with the $\mathrm{FH} 40 \mathrm{G}$ dose rate measuring unit [15].

Figure 2 shows a selection of data collected during one of the measurements. The neutron dose rates, measured by WENDI-2, are normalized to the proton beam current and plotted as a function of time. The solid line corresponds to the average value of the collected dose rates and the four dashed lines indicate the standard deviation and the standard deviation of the average. The average dose rates are used for the comparison to the simulations, which are discussed below. A likely origin for the oscillations observed in the data is related to the low count rate (as calculated from the measured average dose rate and using the sensitivity coefficient described in Sec. IV C) and the time constant of the internal electronics. The calculated standard deviation of 18\%, for the data shown in Fig. 2, closely corresponds to the expected standard deviation for measurements in similar radiation fields, as indicated by the manufacturer [15].

\section{SIMULATIONS AND MODEL}

\section{A. Geant4 simulation toolkit}

Geant4 is a popular Monte-Carlo toolkit for the simulation of the passage of particles through matter [6]. In the present work, it was used to perform the simulations of the particles propagating through the SINQ shielding structures and for the WENDI-2 response function calculations.

The toolkit is developed by a worldwide collaboration of physicists and software engineers, and has a number of attractive features. It has been implemented in the $\mathrm{C}++$ programming language using an open source license. The code is widely used across a number of different scientific fields including high-energy, particle, nuclear, and accelerator physics, as well as studies in medical and space science. It is designed to create and handle complex geometries and can be used for different applications due to the variety of software components available. Experimental data that are present in the toolkit have been drawn from a number of sources around the world. The physics models available include electromagnetic, hadronic, and optical processes and support the transport of particles which are of primary interest for deep-shielding calculations, i.e., neutrons, protons, electrons, and photons. The various implementations which are offered cover a diverse set of interactions and an extended energy range [16]. Of particular importance for spallation neutron physics is the support for theoretical hadronic models for intranuclear transport. Additionally, general-purpose biasing methods have been introduced into the toolkit, including geometrical splitting, Russian roulette, and the weight-window method. This makes it possible to run simulations of large-scale models with numerous components and acquire sufficient statistics in a reasonable time. This is of key importance for radiation shielding investigations and can lead to substantial improvement in the speed of calculations.

\section{B. Simulation methodology}

The Geant 4 simulations of SINQ were performed using a single calculation approach, with the aim being to minimize the number of steps required to go from the generation of primary source particles to the detected neutrons. This approach avoids the difficulties of defining arbitrary source/tally boundaries at various locations throughout the model. The simulations were first carried out using a model of the SINQ, starting with the primary proton beam, and finishing with a score of the neutron fluence and energy spectra at the positions of the neutron dosimeter measurements. The neutron fluence was folded with the simulated response of the WENDI-2 neutron dosimeter, as described in the following section, and normalized to the SINQ proton beam strength by the equation:

$$
D\left[\frac{\mu \mathrm{Sv}}{\mathrm{hr}}\right]=\frac{R\left[\mu \mathrm{Sv} \cdot \mathrm{cm}^{2}\right]}{A\left[\mathrm{~cm}^{2}\right] \times N_{p}^{\text {sim }}[\text { protons }]} \times S\left[\frac{\text { protons }}{\mathrm{hr}}\right],
$$

where $D$ is the dose rate, $R$ the dose response of WENDI-2, $A$ the cross sectional area of the scoring region in the simulation, $N_{p}^{\text {sim }}$ the number of simulated protons on target, and $S$ is the source strength of SINQ. The results presented below are thus an absolute normalization of the simulations for the comparison to the measured data. The calculation of each term will be described in detail in the following sections.

\section{WENDI-2 dosimeter response function}

The absolute response function of the WENDI-2 dosimeter was simulated using Geant4. The calculations were 
carried out for the WENDI-2 dosimeter itself and also for the WENDI-2 dosimeter with a wooden panel, as used to suspend the dosimeter from the crane in the measurements. In the simulations, the wooden panel was modeled as $3 \mathrm{~cm}$ thick with a composition of $6 \%$ hydrogen, $54 \%$ carbon, and $40 \%$ oxygen. The response function was calculated by simulating a full illumination of the side-surface of the dosimeter with a neutron beam and counting the number of tritons produced in the ${ }^{3} \mathrm{He}$ tube. These results were normalized to the number of neutrons incident on the surface of the dosimeter and yielded the absolute response function in units of counts $\cdot \mathrm{cm}^{2}$. A correction factor of 0.743 was included to account for wall effects and a lower discriminator setting in the counting circuitry [17]. An important contribution to the response function is related to the thermal scattering of neutrons in the moderator surrounding the ${ }^{3} \mathrm{He}$ tube, as highlighted in [17]. This contribution was activated in the Geant 4 model for the simulations. The additional physics processes were included as described in the following Sec. IV D.

The response functions are shown in Fig. 3. The two response functions deviate below $10 \mathrm{MeV}$ from each other. In the energy range from $10 \mathrm{MeV}$ to $1 \mathrm{GeV}$, the response functions are identical. The neutron dose response, $\mathrm{R}$ in Eq. (1), was calculated by folding the neutron fluence $\left[\mathrm{cm}^{(-2)}\right]$, simulated as described in the following section, with the absolute response function [counts $\cdot \mathrm{cm}^{2}$ ] in Fig. 3 and by including the sensitivity coefficient $0.84 \mathrm{cps} /(\mu \mathrm{Sv} / \mathrm{h})$, as indicated by the manufacturer of the dosimeter [18]. This procedure yielded the dose response $\mathrm{R}$ in $\mu \mathrm{Sv} \cdot \mathrm{cm}^{2}$. The calibration of the sensitivity coefficient was determined by the manufacturer using a ${ }^{252} \mathrm{Cf}$ source. Thus the measured and simulated dose rates presented in this paper are both based on the calibration of WENDI-2 in this radiation field. A procedure for adjusting

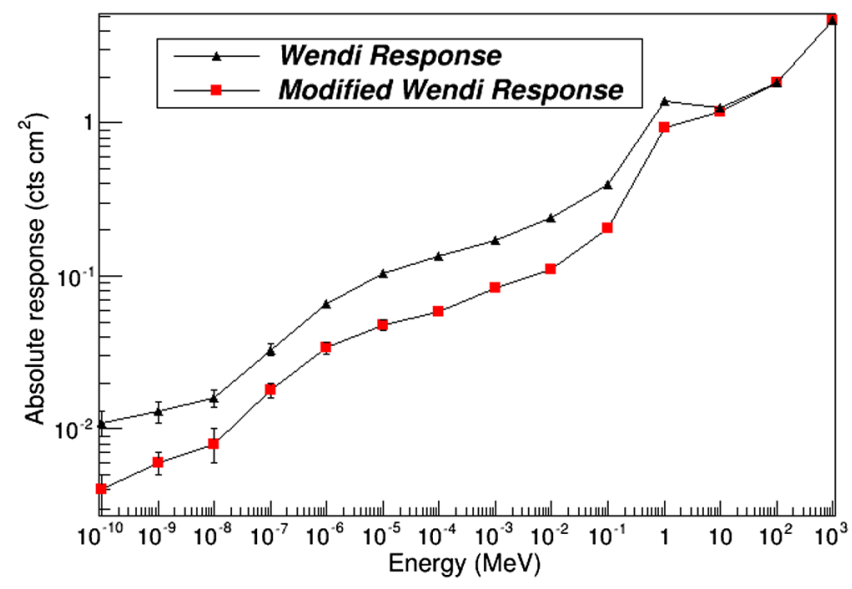

FIG. 3. Geant4 WENDI-2 response function. The black triangle points represent the response function of the dosimeter itself, and the red square points represent the response function of the dosimeter behind the wooden panel.

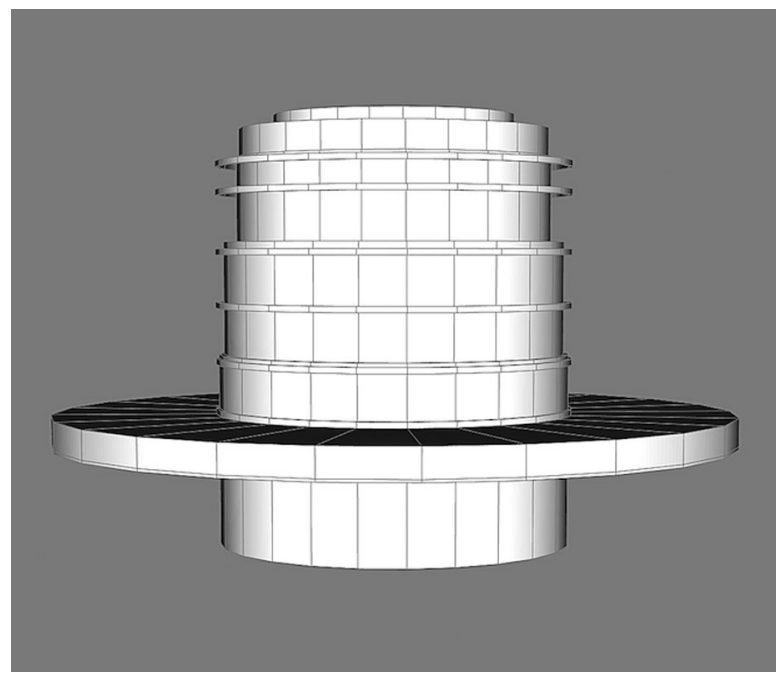

FIG. 4. Model of SINQ monolith in Geant4.

the calibration coefficient to a particular radiation field was outlined in [19]. However, as the aim of this study was to benchmark simulations and measurements, precise determination of the dose rate was not considered a primary goal.

\section{Model of SINQ in Geant4}

A model of the SINQ target station and associated shielding was constructed in Geant 4 . The physics list "QGSP BERT HP" [20], recommended for shielding applications of all energies, was chosen for the simulations. In the abbreviation, "QGS" stands for the quark-gluon string model, which handles the formation of strings in an initial collision of a hadron with a nucleon in the nucleus. "P" means precompound, which is considered an extension of the hadron kinetic model and provides the possibility to extend the low-energy range of the hadron kinetic model for nucleon-nucleus inelastic collisions. "BERT" means that Bertini intra-nuclear cascade model [21] is used. This cascade begins when the incident particle strikes a nucleon in the target nucleus and produces secondaries. The secondaries may interact with other nucleons or be absorbed. Relativistic kinematics is also applied in the model. "HP" states that high-precision neutron tracking model is used to transport neutrons below $20 \mathrm{MeV}$ down to thermal energies [22]. Apart from the standard Geant4 toolkit, the computational framework, developed and maintained by ESS Detector Group [23,24], was used for the simulations. It provides useful data analysis formats and computing cluster parallelization.

A set of variance reduction techniques was implemented in the simulations in order to enhance the computational speed. These techniques are of critical importance, as calculations of the neutron intensities at the positions outside the monolith span approximately 15 orders of magnitude relative to the primary beam intensity. At the source position, 
only neutrons leaving the target-moderator-reflector region with energies above $1 \mathrm{MeV}$ were simulated. Neutrons below this cutoff threshold were discarded at the boundaries of the region. This has been found to be an appropriate assumption in deep-shielding calculations of accelerator-driven spallation sources [4]. Additionally, a parallel world geometry was defined which consisted of a mesh of cells overlaying a section of the mass geometry used in the simulation. This section included the geometrical shapes above the spallation target and moderator region. An importance value was assigned to each cell in such a manner to enhance the propagation of neutrons toward the outer surfaces of the monolith. A total of 132 importance cells were used in the simulation and Russian roulette and geometrical splitting were implemented on the boundaries of the cells. Lastly, cylindrical symmetry in the model and detector geometry was imposed in order to substantially increase the volume of the neutron fluence scoring regions.

Figure 4 shows the model of the SINQ as rendered by Geant4. A detailed description of the spallation target is implemented in the model and consists of 342 lead rods in zircaloy tubes. The geometrical objects in the model, which represent the components of the shielding structure, are approximated by cylinders and tubes. This is for the cylindrical symmetry mentioned previously. The cylindrical components represent the material between the neutron source and the various measurement positions along the outer wall of the monolith. The measurements were performed in a vertical line on top of the BOA instrument cave. The cylinder with the largest radius represents the BOA instrument cave. The thin bands in Fig. 4, that go around the monolith at various positions above the roof, are the perfect sensitive volumes used for scoring the neutrons. The information scored by these volumes was written to a file and contained the particle type and energy. These data were then folded with the WENDI-2 response function, shown in Fig. 3, in order to calculate the simulated neutron radiation dose rate described in Sec. IV C.

It is important to keep in mind that the model is a simplification of some of the monolith's components. This is applicable for both geometrical shapes and material definitions. For example, the storage room was modeled to be empty and filled with air. In reality the room has some equipment, but this is not considered to be an essential detail for the present study. Modeling each component would add orders of magnitude more complexity (and hence simulation time) to the model. Additionally, accurate modeling of inhomogeneities in the materials and gaps between shielding components is challenging as these features are not visible from the outside of the monolith. In order to compensate for the above mentioned differences, the sizes of concrete shielding around the storage room and the layer of borated concrete (outer layer of the monolith) were adjusted. The adjustments correspond to approximately $10 \mathrm{~cm}$ of shielding materials.

\section{RESULTS AND DISCUSSION}

The simulation described in the previous sections took approximately 116 hours on $200 \mathrm{CPU}$ cores to reach satisfactory convergence, and used $7 \times 10^{9}$ primary Monte-Carlo particles (protons) in total. The neutron fluence and the neutron energy spectra calculated from these simulations were converted to neutron dose rates with Eq. (1) as described in Sec. IV B. A plot showing the comparison between the measured and simulated data is presented in Fig. 5. The black squares indicate the measured data and the red triangles represent the simulated data. The data points are given also in Table I, including the absolute errors. The error bars for the simulated values are shown on the graph, whereas the error bars for the measured values are small and hidden by the data point markers. The measured data has also been corrected for the background radiation present at the facility as measured at the distance about $15 \mathrm{~m}$ from the monolith. The points, corresponding to $0.1 \mathrm{~m}, 8.11 \mathrm{~m}$, and $8.89 \mathrm{~m}$, were found to be consistent with these background measurements. A possible origin of the background is related to the leakage from the accelerator, neutron instruments and scattering from the walls of the experimental hall.

The Geant 4 simulations show good agreement with the measured data. For the points where the measured radiation dose is above the background level, the agreement between measurements and simulations is about a factor of 2 . The differences between the trends of the measured and simulated data may be related to the omission of the geometrical details of the model. The inclusion of these details could lead to a significant increase in the required simulation time. However, it can be noted that these differences are of similar magnitude to previous deepshielding measurements/simulations studies with other popular Monte-Carlo codes $[25,26]$. This highlights the suitability of using Geant 4 calculations for shielding design

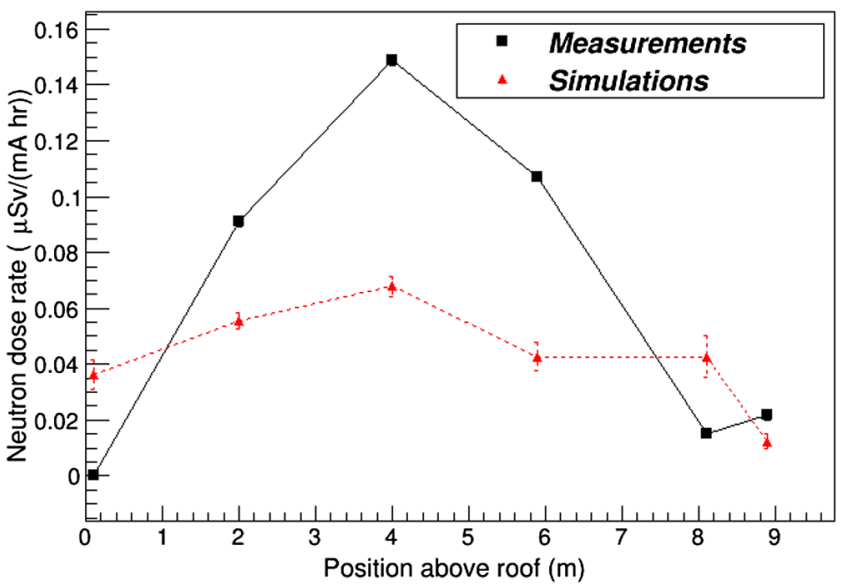

FIG. 5. Comparison between measured data (black squares) and simulated data (red triangles). The lines are a guide for the eye. 
TABLE I. The comparison between measured and simulated dose rates at different positions. Positions are given with respect to BOA roof. The error bars represent the standard errors.

\begin{tabular}{lcc}
\hline \hline Position, $m$ & Measured dose, $\mu \mathrm{Sv} / \mathrm{h}$ & Simulated dose, $\mu \mathrm{Sv} / \mathrm{h}$ \\
\hline 0.10 & $0.000 \pm 0.001$ & $0.036 \pm 0.005$ \\
2.00 & $0.091 \pm 0.002$ & $0.055 \pm 0.003$ \\
4.00 & $0.149 \pm 0.002$ & $0.068 \pm 0.004$ \\
5.89 & $0.107 \pm 0.001$ & $0.043 \pm 0.005$ \\
8.11 & $0.015 \pm 0.001$ & $0.043 \pm 0.007$ \\
8.89 & $0.022 \pm 0.002$ & $0.012 \pm 0.003$ \\
\hline \hline
\end{tabular}

of accelerator-driven spallation sources, and validates the variance reduction and geometry optimization methodologies that were employed.

Table II presents information related to the energy distributions of the neutrons contributing to both the total intensity and the dose rate at each of the measured positions. For each quantity, three groups of data are presented according to their energies, and the fraction of the neutron intensity and of the radiation dose rate induced by neutrons of these energy groups are given, respectively. For the dose rate fractions, the dose conversion factors from ICRP 116 were used [27]. Amongst the neutrons that are escaping from the monolith wall, neutrons with energies below $10 \mathrm{keV}$ make up the majority $(82.7 \%$-96.7\%) of the intensity regardless of position. Neutrons with energies from $10 \mathrm{keV}$ to $10 \mathrm{MeV}$ make up the fractions from $3.3 \%$ to $15.2 \%$. These are neutrons which correspond to the pronounced resonance structure in the cross-section of ${ }^{56} \mathrm{Fe}$. The neutrons with energies above $10 \mathrm{MeV}$, corresponding to a maximum of $8.2 \%$ of the total neutron intensity at a given location, can lead to the creation of the particle showers in the vicinity of instruments detectors. These showers can result in the creation of additional neutrons which can thermalize in shielding materials and ultimately result in signal in instrument detector. Note also that a high-energy component in the intensity is more prevalent in the beam forward directions, i.e., positions further up the monolith wall, as expected from the strong angular dependence of the high-energy neutrons escaping from the target region [4].
Additionally, in the beam forward directions, the dose rates also contain a large component originating from the neutrons above $10 \mathrm{MeV}$. For example, at 8.11 meters $72.7 \%$ of the neutron dose comes from neutrons with energies above $10 \mathrm{MeV}$, amounting to $8.2 \%$ of the total neutron intensity.

In the following, we extrapolate what the simulated fluxes at the edge of the monolith might mean for a neutron scattering instrument at ESS. To observe the highest impact, we investigate an instrument cave at a distance of 25 meters from the monolith, corresponding to the length of a short instrument at ESS. The average simulated flux emitted from the SINQ monolith wall of the neutrons with the energies above $10 \mathrm{MeV}$ was calculated to be about $100 \mathrm{n} /\left(\mathrm{m}^{2} \mathrm{~s}\right)$. The potential presence of neutrons above $10 \mathrm{MeV}$ outside of the shielding is unique for spallation neutron sources compared to reactor-based sources. These energetic neutrons are able to traverse significant distances in air, walls, and light construction material, and depending on their energy either thermalize themselves or create secondary neutrons at the various locations throughout the facility. The sizes of the source and cave used in the extrapolation for ESS were both $25 \mathrm{~m}^{2}$. This corresponds to the approximate area of the bare side of the monolith facing the instrument cave.

Using the source flux of a $100 \mathrm{n} /\left(\mathrm{m}^{2} \mathrm{~s}\right)$, the neutron flux on the outer surface of the cave walls was found to be approximately $0.3 \mathrm{n} /\left(\mathrm{m}^{2} \mathrm{~s}\right)$. Interestingly, this number is of the same order of magnitude as the prompt-pulse background signal at the Cold Neutron Spectrometer (CNCS) [28] at the Spallation Neutron Source in Oak Ridge National Laboratory, Oak Ridge, TN, USA (SNS). This signal is observed when the primary shutter of the instrument is closed which indicates an external source of the neutrons. A reduction by a factor of 100 would make the prompt-pulse signal consistent with the surrounding background level.

The above extrapolation highlights the importance of both the cave design and the monolith design in reducing the prompt-pulse signal. It is important to bear in mind that this is only one part of the prompt-pulse background signal. The other components include skyshine from the proton accelerator, particles streaming down the neutron guides

TABLE II. Data presented in the table show the relative number of neutrons and the dose rates due to these neutrons in a certain energy range calculated at various positions from the simulation results. Positions are given with respect to BOA roof.

\begin{tabular}{|c|c|c|c|c|c|c|}
\hline \multirow[b]{2}{*}{ Position, m } & \multicolumn{2}{|c|}{ Energy $<10 \mathrm{keV}$} & \multicolumn{2}{|c|}{ Energy $10 \mathrm{keV}-10 \mathrm{MeV}$} & \multicolumn{2}{|c|}{ Energy $>10 \mathrm{MeV}$} \\
\hline & Neutrons & Dose & Neutrons & Dose & Neutrons & Dose \\
\hline 0.10 & $84.8 \%$ & $39.5 \%$ & $15.2 \%$ & $60.5 \%$ & $0 \%$ & $0 \%$ \\
\hline 2.00 & $96.7 \%$ & $82.2 \%$ & $3.3 \%$ & $17.8 \%$ & $0 \%$ & $0 \%$ \\
\hline 4.00 & $94.3 \%$ & $55.9 \%$ & $5.4 \%$ & $15.9 \%$ & $0.4 \%$ & $28.2 \%$ \\
\hline 5.89 & $94.0 \%$ & $23.8 \%$ & $4.0 \%$ & $15.2 \%$ & $2.0 \%$ & $61.1 \%$ \\
\hline 8.11 & $86.4 \%$ & $6.6 \%$ & $5.4 \%$ & $20.8 \%$ & $8.2 \%$ & $72.7 \%$ \\
\hline 8.89 & $82.7 \%$ & $10.7 \%$ & $14.5 \%$ & $50.7 \%$ & $2.8 \%$ & $38.6 \%$ \\
\hline
\end{tabular}


and cross-talk from neighboring beamlines. Detailed simulations of the instruments caves and all source components are thus of a critical importance for calculation of the total background on the neutron scattering instruments.

\section{CONCLUSIONS}

In summary, we carried out measurements of the neutron doses rate at various positions along the SINQ monolith. The measurements were compared to a single calculation simulation approach, via an absolute normalization, using the Geant 4 toolkit and yielded reasonable agreement. An agreement between measurements and simulations is about a factor of 2 for the points where the measured radiation dose was above the background level, which is consistent with previous deep-penetration shielding studies carried out with other Monte-Carlo simulation packages. It was found that in the beam forward direction, a significant fraction of the neutron dose rate was related to neutrons with energies above $10 \mathrm{MeV}$, while the total intensity of these neutrons was on the few percent level. It was also shown that the dose at the edges of the monolith could be described by considering only the neutrons leaving the target region with energies above $1 \mathrm{MeV}$.

The current work both extends the availability of experimental data for deep-shielding calculations at accelerator driven spallation sources and supports the fidelity of Geant 4 for such calculations. These findings are important for developing shielding concepts at future accelerator driven spallation neutron sources, particularly the ESS, along with providing benchmarks for popular Monte-Carlo codes.

\section{ACKNOWLEDGMENTS}

One of the authors (G. E.) acknowledges funding by the Scientific User Facilities Division, Office of Basic Energy Sciences, U.S. Department of Energy.

[1] G. J. Russell, H. Robinson, G. L. Legate, and R. Woods, in Proceedings of the ICANS-X Conference, Los Alamos, NM (1988), http://www.neutron.anl.gov/ipns/icans.html.

[2] N. Cherkashyna et al., J. Phys. Conf. Ser. 528, 012013 (2014).

[3] N. Cherkashyna et al., arXiv:1501.02364.

[4] I. Koprivnikar and E. Schachinger, Nucl. Instrum. Methods Phys. Res., Sect. A 487, 571 (2002).
[5] F. Gallmeir, Radiation Protection Dosimetry 115, 23 (2005).

[6] S. Agostinelli et al., Nucl. Instrum. Methods Phys. Res., Sect. A 506, 250 (2003).

[7] S. Peggs et al., ESS Technical Design Report No. ESS2013-001.

[8] http://home.web.cern.ch.

[9] http://www.j-parc.jp/MatLife/en.

[10] http://j-parc.jp/index-e.html.

[11] http://www.psi.ch/sinq.

[12] W. Wagner, SAFERIB Workshop (CERN, Geneva, Switzerland, 2001), p. 15.

[13] R. H. Olsher, H.-H. Hsu, A. Beverding, J. H. Kleck, W. H. Casson, D. G. Vasilik, and R. T. Devine, Health Phys. 79, 170 (2000).

[14] http://www.psi.ch/sinq/boa.

[15] Operating instructions DB-033-961017 E (Thermo Fisher Scientific Messtechnik GmbH, 2012).

[16] J. Allison et al., IEEE Trans. Nucl. Sci. 53, 270 (2006).

[17] T. Vanaudenhove, A. Dubus, and N. Pauly, Radiation Protection Dosimetry 1 (2012).

[18] http://www.thermoscientific.com.

[19] L. Jägerhofer et al., Prog. Nucl. Sci. Technol. 2, 258 (2011).

[20] Geant4 physics reference manual, http://geant4.cern.ch/ G4UsersDocuments/UsersGuides/PhysicsReferenceManual/ html/PhysicsReferenceManual.

[21] H. W. Bertini and M. P. Guthrie, Nucl. Phys. A169, 670 (1971).

[22] P. Speckmayer, Report No. LCD-Note-2010-002.

[23] T. Kittelmann, I. Stefanescu, K. Kanaki, M. Boin, R. Hall-Wilton, and K. Zeitelhack, J. Phys. Conf. Ser. 513, 022017 (2014).

[24] T. Kittelmann and M. Boin, Comput. Phys. Commun. 189, 114 (2015).

[25] M. Sasaki, N. Nakao, T. Nunomiya, T. Nakamura, A. Fukumura, and M. Takada, Nucl. Instrum. Methods Phys. Res., Sect. B 196, 113 (2002).

[26] N. Nakao, T. Nunomiya, H. Iwase, and T. Nakamura, Nucl. Instrum. Methods Phys. Res., Sect. A 530, 379 (2004).

[27] International Commission on Radiological Protection, ICRP Publication 116, Annals of the ICRP $\mathbf{4 0}$ (2010), http://www.icrp.org/publication.asp?id=ICRP\% 20Publication\%20116.

[28] G. Ehlers, A. A. Podlesnyak, J. L. Niedziela, E. B. Iverson, and P. E. Sokol, Rev. Sci. Instrum. 82, 085108 (2011). 